Disponível em

http://www.anpad.org.br/rac

RAC, Rio de Janeiro, v. 17, n. 4, art. 2 , pp. 418-437, Jul./Ago. 2013

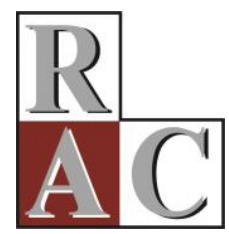

\title{
Antecedentes e Consequências dos Conflitos entre Trabalho e Família
}

\section{Antecedents and Consequences of Work-Family Conflicts}

Lucia Barbosa de Oliveira

E-mail: lucia.oliveira@ibmecrj.br

Faculdades Ibmec-RJ

Av. Pres. Wilson, 118, 20030-020, Rio de Janeiro, RJ, Brasil.

Flávia de Souza Costa Neves Cavazotte

E-mail: flavia.cavazotte@iag.puc-rio.br

Pontifícia Universidade Católica do Rio de Janeiro - PUC-Rio IAG/PUC-Rio, Rua Marquês de São Vicente, 225, 22451-900, Rio de Janeiro, RJ, Brasil.

Raul Ricardo Paciello

E-mail: raulpaciello@uol.com.br

Faculdades Ibmec-RJ

Av. Pres. Wilson, 118, 20030-020, Rio de Janeiro, RJ, Brasil. 


\title{
Resumo
}

A interface entre trabalho e família tem atraído a atenção de pesquisadores ao longo das últimas três décadas (Greenhaus, 2008). Transformações no contexto do trabalho e uma maior preocupação com o equilíbrio entre vida pessoal e vida profissional têm contribuído para ampliar o interesse pelo tema. A partir de uma amostra de 296 trabalhadores com nível superior, foram testadas hipóteses formuladas com base no modelo proposto por Anderson, Coffey e Byerly (2002). Em primeiro lugar, avaliaram-se os impactos de políticas organizacionais (flexibilidade de horário e apoio no cuidado com dependentes) e de práticas informais (suporte gerencial e percepção de consequências na carreira) sobre o conflito trabalho-família (CTF) e o conflito família-trabalho (CFT). Também foram testadas hipóteses relativas às consequências desses conflitos, especificamente satisfação no trabalho, intenção de deixar a empresa, estresse e absenteísmo. Os resultados indicaram que o suporte gerencial contribui para reduzir o CTF, enquanto que a percepção de consequências na carreira tende a promovêlo. Com relação às consequências dos conflitos, foram encontrados impactos significativos sobre a satisfação no trabalho, o estresse e a intenção de deixar a empresa, mas não sobre o absenteísmo.

Palavras-chave: conflito trabalho-família; qualidade de vida no trabalho; estresse; intenção de deixar a empresa; políticas de recursos humanos.

\begin{abstract}
The work-family interface has attracted the attention of researchers over the past three decades (Greenhaus, 2008). Changes in the world of work and a growing concern about the balance between personal and professional lives have contributed to increased interest in the topic. Drawing on a sample of 296 workers with higher education, hypotheses were tested based on the model proposed by Anderson, Coffey and Byerly (2002). First, we evaluated the impact organizational policies (schedule flexibility and dependent care benefits) and informal practices (manager support and perceived career consequences) have on the work-to-family conflict (WFC) and family-to-work conflict (FWC). We also tested hypotheses concerning the consequences of these conflicts, specifically job satisfaction, turnover intentions, stress and absenteeism. The results indicated that manager support helps reduce WFC, whereas perceived career consequences tend to promote it. Regarding the consequences of such conflicts, we found significant impacts on job satisfaction, stress and turnover intentions, but not on absenteeism.
\end{abstract}

Key words: work-family conflict; quality of work life; stress; turnover intentions; human resources policies. 


\section{Introdução}

A intensificação do tempo de trabalho e as crescentes demandas por resultados têm exercido fortes pressões sobre a força de trabalho (Burke, 2009; Cappelli, 1999; Pochmann, 2002; Rosso, 2008), ampliando as discussões a respeito dos impactos sobre sua saúde e bem-estar, o que inclui a questão do equilíbrio entre vida pessoal e vida profissional (Eby, Casper, Lockwood, Bourdeaux, \& Brinley, 2005; Greenhaus, 2008; Greenhaus, Collins, \& Shaw, 2003; Lewis \& Cooper, 1999). Mudanças na estrutura demográfica da população, incluindo a maior inserção da mulher no mercado de trabalho (Araújo \& Scalon, 2005), o aumento do número de casais em que ambos trabalham e buscam investir em suas carreiras (Santos \& Casado, 2011), além do envelhecimento da população (Beauregard \& Henry, 2009), que implica em maiores responsabilidades em relação ao cuidado com idosos (Premeaux, Adkins, \& Mossholder, 2007), também têm suscitado pesquisas voltadas a investigar a interface entre os domínios trabalho e família. Contribui ainda para esse problema a disseminação de inovações tecnológicas, especialmente tecnologias de comunicação móvel, que permitem ao trabalhador estar permanentemente disponível para atender a demandas profissionais (Cavazotte, Brollo, \& Moreno, 2009; Chesley, 2005).

Nesse sentido, o conflito entre vida pessoal e vida profissional tem recebido destaque, especialmente na literatura internacional. Cabe registrar que este pode ter origem tanto no universo do trabalho, quando responsabilidades profissionais interferem no cumprimento de obrigações familiares (conflito trabalho-família), quanto no domínio da vida pessoal, quando obrigações relacionadas à família prejudicam o desempenho no trabalho (conflito família-trabalho). Dessa forma, consideram-se o conflito trabalho-família e o conflito família-trabalho como dois construtos diferentes, mas interrelacionados (Anderson, Coffey, \& Byerly, 2002; Beauregard \& Henry, 2009; Greenhaus \& Beutell, 1985).

As organizações também têm se debruçado sobre o problema, na medida em que a vivência desses conflitos pode afetar o desempenho dos empregados e, consequentemente, os resultados da empresa (Beauregard \& Henry, 2009; Yasbek, 2004). Segundo Beauregard e Henry (2009), tem crescido a pressão pela implantação de políticas de apoio e de qualidade de vida no trabalho, com o objetivo de facilitar o esforço dos trabalhadores em cumprir suas responsabilidades profissionais e pessoais.

A fim de contribuir para o conhecimento acumulado nesse campo, o presente estudo, de caráter quantitativo, investigou antecedentes e consequências dos conflitos entre vida pessoal e vida profissional em um grupo de 296 profissionais com formação superior, que atuam em empresas públicas e privadas de diferentes setores da economia. Mais especificamente, procurou-se avaliar, em primeiro lugar, os impactos de políticas formais e práticas informais existentes nas empresas sobre o conflito trabalho-família (CTF) e o conflito família-trabalho (CFT). Dentre as políticas formais, foram consideradas a flexibilidade de horário e as políticas de apoio ao cuidado com dependentes, enquanto que as práticas informais levaram em conta o apoio recebido do superior imediato e a percepção de que a busca por uma vida mais equilibrada implica em consequências negativas para a carreira. Também foram testadas hipóteses relativas aos efeitos desses conflitos sobre a satisfação no trabalho, a intenção de deixar a empresa, o estresse e o absenteísmo.

O entendimento da dinâmica dos conflitos entre vida pessoal e profissional pode auxiliar as organizações no desenho de políticas e no fomento de práticas voltadas ao equilíbrio entre esses dois universos, permitindo maior eficiência na aplicação de recursos e a criação de um ambiente organizacional mais estável e produtivo, o que confere relevância ao presente trabalho. Também cabe ressaltar que, na literatura nacional, há poucos estudos quantitativos dedicados à compreensão dessa relação entre trabalho e família (e.g. Furtado, 2012; Tanure, Carvalho, \& Andrade, 2007). Além disso, o entendimento das consequências de tais conflitos sobre o trabalhador pode contribuir para chamar a atenção a uma tendência que parece estar cada vez mais disseminada no universo corporativo 
brasileiro e que pode, a médio e longo prazos, gerar consequências nocivas tanto para os trabalhadores como para o desempenho das organizações.

\section{Referencial Teórico}

A fundamentação teórica para a pesquisa empírica aqui proposta é apresentada a seguir. Começa-se com uma breve discussão sobre o conceito de qualidade de vida no trabalho e suas dimensões, entre as quais se inclui o equilíbrio entre o trabalho e as demais esferas da vida. Em seguida, parte-se para o tema da interface entre vida pessoal e vida profissional, em que são abordadas as diferentes perspectivas sobre essa relação, com destaque para o problema do conflito entre trabalho e família. Discute-se a evolução do conceito e suas interpretações, as políticas e as práticas que visam sua minimização e também as consequências investigadas na literatura.

\section{Qualidade de vida no trabalho}

O conceito de qualidade de vida no trabalho (QVT) tem sido alvo de múltiplas interpretações, tendo estas alguns pontos em comum, como a ideia da humanização do trabalho e a preocupação com a satisfação e o bem-estar dos trabalhadores (Sant'Anna \& Kilimnik, 2011). Ferreira, Ferreira, Antloga e Bergamaschi (2009) distinguem duas abordagens para a qualidade de vida no trabalho: a assistencialista e a preventiva. Segundo os autores, grande parte dos estudos se alinham à primeira corrente, caracterizada pela lógica da compensação, pela responsabilização do trabalhador por sua qualidade de vida e pelo foco na produtividade. Já a abordagem preventiva, que surge como "uma concepção contra-hegemônica de QVT", concentra-se na remoção dos fatores causadores de mal-estar no trabalho e no entendimento de que a produtividade seria uma consequência do processo (Ferreira, Ferreira, Antloga, \& Bergamaschi, 2009, p. 149).

As dimensões do conceito de QVT também têm sido tratadas de diferentes formas. Segurança, nível de estresse, equilíbrio trabalho-família, estilo de gestão e supervisão, jornada de trabalho, ambiente físico, aspectos financeiros, grau de autonomia, entre outros, vêm sendo associados ao construto (Bagtasos, 2011). O modelo de Walton (1973), um dos mais abordados na literatura (Ferreira et al., 2009), considera oito indicadores: compensação justa e adequada, condições de trabalho, oportunidade de uso e desenvolvimento das capacidades, carreira e segurança, integração social, constitucionalismo, relevância social e relação entre trabalho e espaço total da vida, esta última foco do presente estudo e discutida a seguir.

\section{Interface entre trabalho e família}

Mudanças no mundo do trabalho, amplamente discutidas por Cappelli (1999) e Pochmann (2002), entre outros, têm ampliado as exigências sobre os trabalhadores (Burke, 2009) e gerado impactos sobre a qualidade de vida no trabalho. Isso inclui o problema do conflito trabalho-família, que ocorreria quando o excesso de demandas oriundas do trabalho dificultaria o exercício de papéis pessoais, tais como os cuidados com os filhos e outros dependentes. As mudanças observadas na estrutura das famílias, por sua vez, vêm contribuindo para o problema do conflito família-trabalho, observado quando as responsabilidades familiares prejudicam o desempenho no trabalho (Carr, Boyar, \& Gregory, 2008; Premeaux et al., 2007). No passado, a estrutura familiar clássica era composta pela figura do homem provedor e pela mulher que se dedicava às tarefas do lar. Atualmente, verifica-se a maior participação de mulheres na força de trabalho, mais famílias monoparentais (Araújo \& Scalon, 2005) e mais pessoas com responsabilidades de cuidar de pais idosos (Premeaux et al., 2007). Essa menor separação entre papéis profissionais e pessoais implicaria numa maior possibilidade de ocorrência de conflitos entre esses dois universos. 
$\mathrm{Na}$ literatura sobre o tema, porém, observam-se duas perspectivas para a interface entre trabalho e família: a do esgotamento, focada na questão do conflito de papéis, e a facilitadora (Bagger, Li, \& Gutek, 2008), também chamada de enriquecedora (Greenhaus \& Powell, 2006). De acordo com a primeira perspectiva, as demandas relativas aos papéis desempenhados no trabalho e na família seriam, de alguma forma, incompatíveis (Carr et al., 2008; Greenhaus \& Beutell, 1985). Em outras palavras, o conflito ocorreria quando responsabilidades associadas a um domínio prejudicassem o desempenho de funções e atividades do outro. Greenhaus e Beutell (1985) identificaram três principais formas de ocorrência desse conflito: (a) tempo, em que múltiplos papéis competiriam pelo tempo da pessoa; (b) tensão, quando pressões vividas num domínio provocariam fadiga ou irritabilidade, afetando o desempenho do outro papel; e (c) comportamento, quando as expectativas de conduta associadas a um papel seriam incompatíveis com o comportamento esperado no outro. Por exemplo, os estereótipos da objetividade e da agressividade associados ao ambiente de trabalho podem não ser adequados ao espaço familiar e, se o indivíduo não for capaz de se ajustar aos diferentes papéis, pode experimentar conflito. De acordo com a perspectiva enriquecedora, que vem recebendo crescente atenção na literatura, experiências e habilidades desenvolvidas num domínio poderiam ajudar no desempenho de atividades no outro (Greenhaus \& Powell, 2006; Powell \& Greenhaus, 2010). A presente pesquisa teve por objetivo contribuir para a literatura do primeiro grupo de estudos.

Nos trabalhos que avaliam a questão sob a perspectiva do conflito de papéis, um tema importante refere-se à saliência de papéis, ou seja, à importância atribuída a um ou a outro papel (de trabalhador ou de mãe/pai de família), que tenderia a interferir na forma como os conflitos são vividos. Carr, Boyar e Gregory (2008), por exemplo, encontraram evidências de que a relação entre conflito trabalho-família e atitudes relacionadas ao trabalho (satisfação no trabalho e comprometimento organizacional) é moderada pela importância do trabalho na vida. De forma análoga, Bagger, Li e Gutek (2008) obtiveram suporte às hipóteses de que a saliência da identidade familiar intensifica o efeito do conflito família-trabalho sobre o sofrimento no trabalho e a satisfação no trabalho.

Ainda com relação ao conflito entre trabalho e família, pesquisas têm abordado o conceito de duas formas distintas. Inicialmente, considerava-se uma relação não direcional de interferência entre trabalho e família (Eby et al., 2005). Mais recentemente, porém, tem se assumido a bidirecionalidade do conceito, em que conflito trabalho-família e conflito família-trabalho seriam, na realidade, construtos distintos, que se influenciam mutuamente e que possuem diferentes antecedentes e consequências (Anderson et al., 2002; Eby et al., 2005). A pesquisa aqui relatada se alinha à segunda abordagem.

Tendo em vista o entrelaçamento dos domínios trabalho e família observados na literatura nacional e internacional, e suas implicações para o bem-estar dos trabalhadores e seus familiares (e.g.: Eby et al., 2005; Oltramari, Grisci, \& Weber, 2011; Tanure et al., 2007), é importante aprofundar o conhecimento sobre o fenômeno. Reconhecendo a existência dos conflitos entre trabalho e família, e as possíveis consequências sobre o bem-estar e o desempenho de seus empregados, as organizações vêm desenvolvendo iniciativas visando evitar ou atenuar tais conflitos. São as políticas de qualidade de vida no trabalho, discutidas a seguir.

\section{Políticas de qualidade de vida no trabalho}

De acordo com Albuquerque e Limongi-França (1998, p. 41), tem crescido no ambiente empresarial brasileiro a preocupação com a qualidade de vida no trabalho, entendida como um conjunto de ações envolvendo "diagnóstico e implantação de melhorias e inovações gerenciais, tecnológicas e estruturais dentro e fora do ambiente de trabalho, visando propiciar condições plenas de desenvolvimento humano para e durante a realização do trabalho". No entanto, os autores destacam que as iniciativas desenvolvidas têm um caráter predominantemente operacional e ainda estão pouco alinhadas à estratégia de recursos humanos das organizações.

Sant'Anna e Kilimnik (2011) ressaltam que uma política de QVT deve ter por objetivo a criação de uma situação mais favorável à satisfação dos empregados, ao mesmo tempo em que fomenta o 
aumento da produtividade. Oliveira e Limongi-França (2005), por sua vez, reconhecem que todas as ações desenvolvidas pela empresa e pelos funcionários que busquem a integração biopsicossocial e o controle dos fatores de risco ocupacionais estão ligadas à gestão da qualidade de vida no trabalho.

Especificamente em relação às políticas de equilíbrio entre vida pessoal e vida profissional (work-life balance practices ou family-friendly policies), Beauregard e Henry (2009) apontam que tais iniciativas usualmente referem-se ao suporte que a organização dá aos cuidados com dependentes, às opções de flexibilidade no trabalho (horário flexível, por exemplo), e à licença para tratar de assuntos pessoais ou familiares. Segundo os autores, essas políticas tipicamente têm por objetivo não apenas reduzir o conflito vivido pelos empregados, mas também são adotadas como estratégia de recrutamento, ao tornar a empresa mais atraente a potenciais trabalhadores.

Com relação à eficácia dessas políticas, os resultados têm sido divergentes. Anderson, Coffey e Byerly (2002), por exemplo, encontraram evidências de que horário flexível impacta negativamente o conflito trabalho-família. No entanto, com relação à disponibilidade e ao uso de políticas de apoio aos dependentes, Premeaux, Adkins e Mossholder (2007) reportaram que a disponibilidade de tais políticas não teve efeito sobre os conflitos trabalho-família e família-trabalho. Resultado semelhante foi verificado por Anderson et al. (2002), que não encontraram confirmação de que o conflito famíliatrabalho fosse afetado pelo uso dessas políticas.

Além das políticas formais, também se discute o impacto de práticas informais - tais como o suporte gerencial e os aspectos relacionados ao contexto e à cultura organizacional - sobre o conflito (Anderson et al., 2002; Eby et al., 2005; Premeaux et al., 2007). A inclusão do suporte gerencial devese ao entendimento de que o superior imediato tende a exercer papel importante, fazendo com que o uso dos benefícios disponíveis e a vivência do conflito sejam diferentes entre membros de uma mesma organização e contingente às crenças e práticas do gestor. McCarthy, Darcy e Grady (2010) destacam que a forma como políticas de recursos humanos são interpretadas, gerenciadas e implementadas dependem, em grande medida, da postura de gerentes médios e de linha. Com relação às variáveis contextuais, Anderson et al. (2002) sugerem que a cultura organizacional pode ser mais ou menos compreensiva e receptiva aos dilemas entre trabalho e vida pessoal. Uma dessas variáveis corresponde às consequências na carreira, em que o empregado percebe a necessidade de sacrificar suas perspectivas de carreira e chances de promoção, caso opte por maior flexibilidade ou por uma vida mais equilibrada (Anderson et al., 2002; Premeaux et al., 2007).

A partir dessas questões levantadas na literatura e com base no modelo desenvolvido por Anderson et al. (2002), são propostas as seguintes hipóteses.

Hipótese 1: (a) quanto mais flexível o horário de trabalho do empregado, (b) quanto maior o suporte gerencial recebido, (c) quanto menor sua percepção de consequências na carreira, e (d) quanto menor o conflito família-trabalho (CFT), menor será o conflito trabalho-família (CTF).

Hipótese 2: (a) quanto maior o número de políticas de apoio ao cuidado com dependentes utilizadas pelo empregado, (b) quanto mais simples for sua estrutura familiar, e (c) quanto menor o conflito trabalho-família (CTF), menor será o conflito família-trabalho (CFT).

\section{Consequências dos conflitos entre trabalho e família}

Além dos antecedentes, as consequências dos conflitos entre trabalho e família também têm sido estudadas (e.g. Anderson et al., 2002; Bagger et al., 2008; Carr et al., 2008; Premeaux et al., 2007). Eby et al. (2005) organizaram os impactos do conflito trabalho-família em três grupos. O primeiro trata de aspectos físicos e psicológicos, tais como estresse, depressão, hipertensão, ansiedade, transtornos de humor e abuso de substâncias. O segundo envolve impactos sobre atitudes e comportamentos do trabalhador, incluindo satisfação no trabalho, comprometimento organizacional e intenção de deixar a empresa. Por fim, o terceiro grupo considera os efeitos sobre a satisfação com a família. 
Premeaux et al. (2007) testaram o efeitos dos conflitos trabalho-família e família-trabalho sobre a satisfação no trabalho, e os comprometimentos afetivo e instrumental, tendo encontrado evidências dessas relações. Bagger et al. (2008), por sua vez, reportaram uma correlação positiva entre conflito família-trabalho e sofrimento psicológico e uma correlação negativa entre esse tipo de conflito e a satisfação no trabalho. Num outro estudo, Carr et al. (2008) obtiveram suporte às hipóteses relativas ao impacto do conflito trabalho-família sobre satisfação no trabalho, comprometimento organizacional e retenção. No presente estudo, novamente com base no modelo de Anderson et al. (2002), são propostas as seguintes hipóteses a respeito das consequências dos conflitos entre trabalho e família.

Hipótese 3: quanto maior o conflito trabalho-família (CTF), menor será a satisfação no trabalho.

Hipótese 4: quanto maior o conflito trabalho-família (CTF), maior será a intenção de deixar a empresa.

Hipótese 5: quanto maior (a) o conflito trabalho-família (CTF) e (b) o conflito família-trabalho (CFT), maior será o estresse.

Hipótese 6: quanto maior o conflito família-trabalho (CFT), maior será o absenteísmo.

A Figura 1 destaca cada uma das hipóteses testadas.

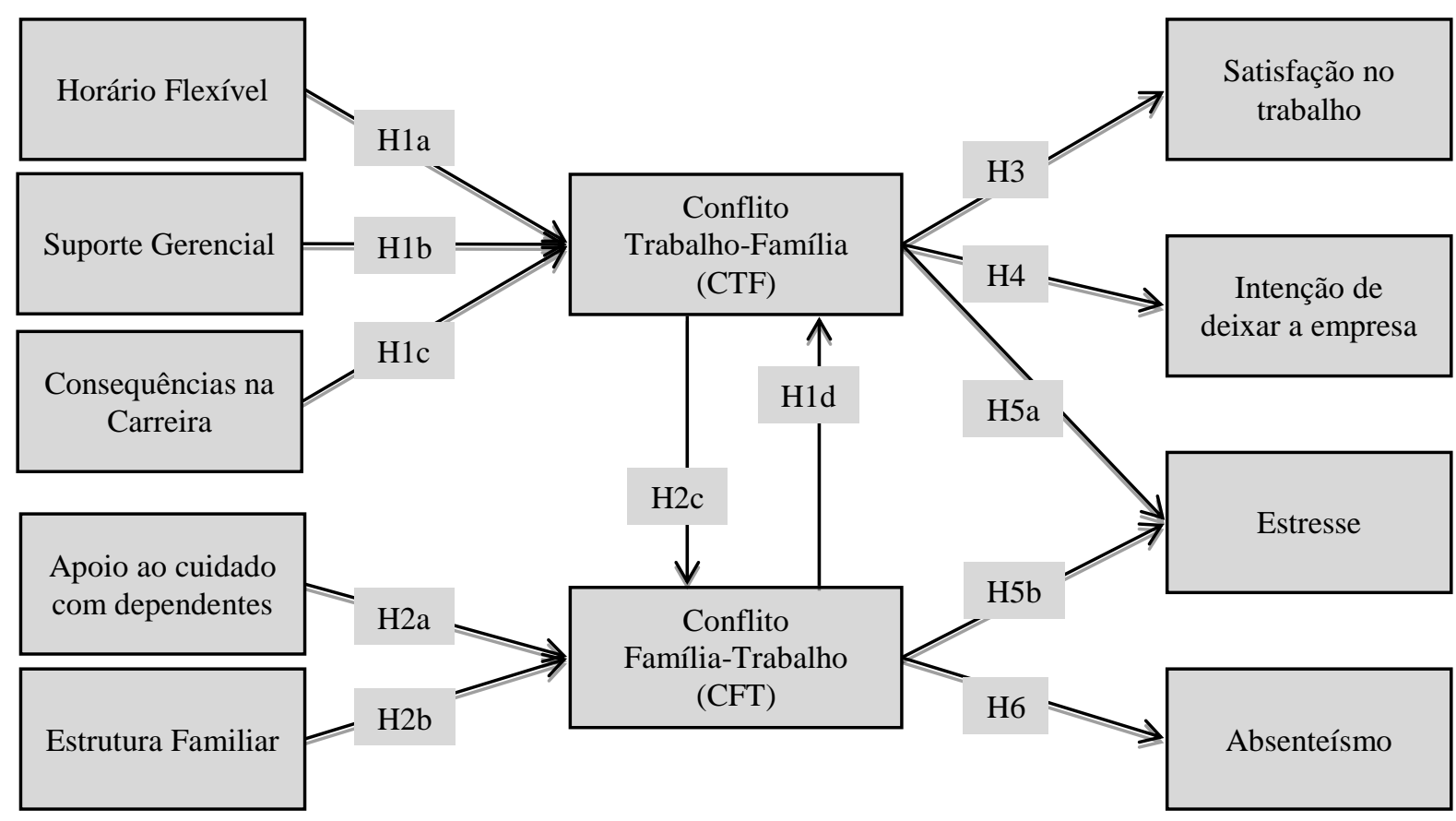

Figura 1. Hipóteses.

\section{Método}

\section{Procedimento e coleta de dados}

A amostra utilizada neste estudo, selecionada por conveniência, foi composta de profissionais com, no mínimo, nível superior completo, vinculados direta ou indiretamente a uma escola de negócios privada do Rio de Janeiro. A coleta de dados foi realizada por meio de um questionário eletrônico armazenado no SurveyMonkey®, e os convites para participar da pesquisa foram enviados 
por e-mail para aproximadamente 2.000 profissionais. No total, 401 participantes em potencial acessaram o questionário (20\%). Desses, 41 abandonaram a pesquisa antes de concluí-la. Foram descartados questionários de indivíduos que não estavam empregados (50), ou que trabalhavam em tempo parcial (14), tendo em vista o propósito do estudo. A amostra final totalizou 296 questionários válidos.

Os participantes da pesquisa atuam majoritariamente em empresas privadas $(82 \%)$ de grande porte $(76 \%)$, de diferentes setores de atividade. A grande maioria (79\%) possui MBA ou mestrado completo, e $63 \%$ ocupam cargos de chefia. A idade dos participantes variou de 22 a 62 anos, sendo a média, 39 anos. Do total de participantes, $54 \%$ são homens, $58 \%$ são casados e $58 \%$ possuem pelo menos um dependente (filhos menores de 18 anos, idosos ou pessoas com necessidades especiais).

\section{Medidas}

As medidas utilizadas basearam-se no trabalho de Anderson et al. (2002), com algumas adaptações, conforme descrito a seguir.

Horário flexível. Foi utilizada a escala de Likert de 5 pontos com três afirmações que buscavam captar o grau de flexibilidade de horário: (a) possibilidade de adoção de esquemas de horários prédefinidos; (b) livre escolha de horário de trabalho; e (c) existência de horário de trabalho completamente rígido (invertido). Os itens foram definidos a partir da pesquisa de Anderson et al. (2002), com pequenas adaptações para facilitar o entendimento.

Apoio ao cuidado com dependentes. Para medir as políticas de apoio ao cuidado com dependentes, considerou-se o número de benefícios utilizados (Anderson et al., 2002; Judge \& Colquitt, 2004). Aos profissionais cujas empresas não ofereciam nenhum tipo de apoio, foi atribuído valor zero. O questionário também continha uma pergunta aberta que pedia ao participante que informasse quais eram os benefícios utilizados.

Suporte Gerencial. Foi utilizada a escala Likert de 5 pontos, com seis itens que abordam o suporte recebido do supervisor imediato quando o empregado busca equilibrar responsabilidades no trabalho e na família, conforme Anderson et al. (2002). São exemplos de itens que compõem essa escala: (a) sinto-me confortável em trazer meus problemas pessoais ou familiares ao conhecimento do meu gerente/supervisor; (b) meu gerente/supervisor é compreensivo quando falo de questões e problemas pessoais ou familiares que afetam meu trabalho.

Consequências na Carreira. Também de acordo com Anderson et al. (2002), foi utilizada a escala Likert de 5 pontos, com cinco afirmações para avaliar a percepção dos respondentes sobre possíveis impactos em suas carreiras caso optassem por usar benefícios oferecidos ou buscassem um maior equilíbrio entre trabalho e família. Um exemplo de item dessa escala é: no meu local de trabalho, os empregados que colocam as necessidades familiares ou pessoais à frente das necessidades do trabalho não são vistos com bons olhos.

Estrutura Familiar. Neste item, avaliou-se a complexidade da estrutura familiar, incluindo a presença de cônjuge, se o mesmo exerce trabalho remunerado, além da existência de dependentes filhos menores de 18 anos, idosos maiores de 65 anos, crianças com necessidades especiais e adultos (não idosos) com necessidades especiais. Atribuiu-se valor zero às respostas negativas e valor um às afirmativas. A variável foi calculada a partir da soma desses valores, podendo variar entre zero e seis (Anderson et al., 2002).

Conflito Trabalho-Família. A partir de cinco questões, buscou-se avaliar a frequência com que o respondente vivenciou esse conflito nos últimos três meses. Falta de tempo, energia, capacidade e humor para resolver assuntos pessoais ou familiares, em decorrência de assuntos relacionados ao trabalho, foram pontos questionados. Foi adotada uma escala de 5 pontos, variando entre nunca e muitas vezes (Anderson et al., 2002). Um exemplo de item dessa escala é: nos últimos três meses, com 
que frequência você não teve energia para fazer coisas com sua família ou outras pessoas importantes por causa do seu trabalho?

Conflito Família-Trabalho. Da mesma forma que para o CTF, cinco itens avaliaram a frequência com que este conflito havia sido vivenciado nos últimos três meses. As questões envolveram até que ponto demandas originadas na família ou na vida pessoal impactaram o desempenho no trabalho. Foi adotada uma escala de 5 pontos, variando entre nunca e muitas vezes (Anderson et al., 2002). Um exemplo de item dessa escala é: nos últimos três meses, com que frequência sua família ou sua vida pessoal impediu você de ter a energia que necessitava para fazer o seu trabalho?.

Satisfação no Trabalho. A satisfação no trabalho foi medida através da aplicação de uma versão resumida e adaptada da Escala de Satisfação no Trabalho proposta por Siqueira (2008). São exemplos de itens que compõem essa escala: (a) gosto de estar com as pessoas com quem trabalho todos os dias; (b) recebo uma remuneração justa pelo trabalho que realizo. Foi adotada a escala Likert de 5 pontos.

Intenção de Deixar a Empresa. Para medir essa variável, foi utilizada uma afirmação que buscou avaliar a intenção do respondente de deixar a empresa nos doze meses seguintes à aplicação da pesquisa. Também foi adotada a escala Likert de 5 pontos.

Estresse. Sete questões mediram o estresse vivido nos últimos três meses, a partir de uma escala de 5 pontos, variando entre nunca e muitas vezes. Foi pedido ao participante que avaliasse aspectos de desequilíbrio orgânico e mental, e a sensação de incapacidade para realizar tarefas (Anderson et al., 2002). Um exemplo de item dessa escala é: durante os últimos três meses, com que frequência você se sentiu cansado quando acordou pela manhã e teve que enfrentar mais um dia de trabalho?

Absenteísmo. Também de acordo com Anderson et al. (2002), foi perguntado aos respondentes quantas vezes, nos últimos três meses, ausentaram-se do trabalho por causa de filhos ou dependentes, ou por outros motivos de natureza familiar.

Para o teste das hipóteses, foram incluídas as seguintes variáveis de controle: sexo $(0=$ mulheres, $1=$ homens), idade, cargo de chefia $(0=$ não, $1=\operatorname{sim})$, tempo de empresa (cinco faixas em ordem crescente de grandeza), tamanho da empresa ( $0=$ até 49 funcionários [pequena], $1=$ de 50 até 99 [média], e 2=100 ou mais funcionários [grande]) e tipo de empresa ( $0=$ pública, $1=$ privada).

\section{Resultados}

Este estudo tem como objetivo principal verificar a adequação do modelo de Anderson et al. (2002) à realidade brasileira. Naquele trabalho, os autores utilizaram a modelagem de equações estruturais para testar suas hipóteses numa amostra de 2877 trabalhadores. Esta técnica é ideal quando se deseja verificar múltiplas relações de dependência entre variáveis. Porém, em função do número de parâmetros a serem estimados e das premissas em máxima verossimilhança (maximum likelihood), o tamanho da amostra torna-se um importante critério de decisão na adoção dessa estratégia de análise (Hair, Anderson, Tatham, \& Black, 1998; Mcquitty, 2004). Como a amostra obtida neste estudo ( $\mathrm{N}=296)$ foi menor do que o mínimo necessário de observações para estimar adequadamente todos os parâmetros envolvidos no modelo, optou-se por empregar a análise de regressão hierárquica, que envolve técnicas estabelecidas e amplamente utilizadas em pesquisas nas ciências sociais e comportamentais (Tabachnick \& Fidell, 2007).

Os dados foram analisados no software estatístico PASW Statistics (antigo SPSS). Quanto às escalas com múltiplos itens, foram calculadas médias que correspondem aos construtos latentes prospectados. Também foram realizadas análises de confiabilidade das escalas utilizadas no estudo. A Tabela 1 apresenta as médias, desvios-padrão e correlações entre as variáveis pesquisadas. Os 
resultados de alfas de Cronbach para todas as escalas se mostraram adequados e foram inseridos na diagonal da Tabela 1.

Tabela 1

Médias, Desvios-Padrão e Correlações

\begin{tabular}{|c|c|c|c|c|c|c|c|c|c|c|c|c|c|}
\hline Variável & Média & DP & 1 & 2 & 3 & 4 & 5 & 6 & 7 & 8 & 9 & 10 & 11 \\
\hline 1. Horário flexível & 3,26 & 1,27 & $(, 73)$ & & & & & & & & & & \\
\hline 2. Políticas de apoio & 0,43 & 0,85 &, 04 & - & & & & & & & & & \\
\hline 3. Suporte Gerencial & 3,88 & 0,89 &, $18^{* * *}$ & ,08 & $(, 86)$ & & & & & & & & \\
\hline 4. Consequências na carreira & 2,51 & 0,98 &,$- 19 * *$ &,- 03 &,$- 49 * *$ & $(, 82)$ & & & & & & & \\
\hline 5. Estrutura Familiar & 1,78 & 1,33 &, $15^{*}$ &, $15^{*}$ &, 07 & ,03 & - & & & & & & \\
\hline 6. CTF & 3,03 & 1,06 &,- 07 &,- 06 &,$- 33 * *$ &, $34 * *$ &, 07 & $(, 90)$ & & & & & \\
\hline 7. CFT & 1,75 & 0,88 &, 04 &, 04 &,- 09 &, $13 *$ & ,06 &, $23 * *$ & $(, 90)$ & & & & \\
\hline 8. Satisfação no trabalho & 4,02 & 0,72 &, $24 * *$ &, 06 &, $62 * *$ &,$- 38 * *$ &, $17 * *$ &,$- 22 * *$ &,- 05 & $(, 75)$ & & & \\
\hline 9. Intenção de deixar & 2,84 & 1,49 &,$- 18^{* *}$ &,$- 14 *$ &,$- 38 * *$ &, $26^{* *}$ &,$- 14^{*}$ &, $24 * *$ &, 08 &,$- 59 * *$ & - & & \\
\hline 10. Estresse & 3,14 & 1,11 &,- 11 &,- 10 &,$- 31 * *$ &, $30 * *$ &, 00 &, $63^{* *}$ &, $30 * *$ &,$- 35 * *$ &, $31^{* *}$ & $(, 92)$ & \\
\hline 11. Absenteísmo & 1,44 & 4,13 &,$- 15^{* *}$ &, 01 &,$- 14 *$ &, 07 &,- 03 &, 11 &, 10 &,$- 23 * *$ &, $13 *$ &, $15^{* * *}$ & - \\
\hline 12. Idade & 39,14 & 9,51 &, 10 & ,09 &, 05 &,$- 12 *$ &, $33^{* *}$ &,- 05 &,- 11 &, $16^{* *}$ &,$- 19 * *$ &,$- 18 * *$ &,- 05 \\
\hline
\end{tabular}

Nota. Valores na diagonal entre parênteses correspondem aos alfas de Cronbach.

$* \mathrm{p}<0,05 * * \mathrm{p}<0,01$.

Foram realizadas análises da variância (ANOVAS) para explorar o efeito das variáveis demográficas categóricas (controles) sobre as variáveis dependentes em estudo. A Tabela 2 apresenta os resultados dessas análises. Conforme se pode observar, as variáveis sexo e tamanho de empresa não tiveram efeitos significativos sobre nenhuma variável substantiva; indivíduos em cargos de chefia indicaram ter níveis maiores de conflito trabalho-família; funcionários com dez ou mais anos de empresa e aqueles que trabalham em empresas públicas apresentaram níveis menores de intenção de deixar a empresa; indivíduos que ocupam cargos de chefia e trabalham em empresas privadas tendem a vivenciar maiores níveis de estresse; e empregados de empresas públicas declararam faltar mais ao trabalho.

Tabela 2

Resumo dos Resultados das ANOVAS

\begin{tabular}{l|ccccc}
\hline Variáveis & Sexo & $\begin{array}{c}\text { Cargo de } \\
\text { Chefia }\end{array}$ & $\begin{array}{c}\text { Tempo de } \\
\text { empresa }\end{array}$ & $\begin{array}{c}\text { Tamanho } \\
\text { da empresa }\end{array}$ & $\begin{array}{c}\text { Tipo de } \\
\text { empresa }\end{array}$ \\
\hline CTF & $\mathrm{ns}$ & $5,29 *$ & $\mathrm{~ns}$ & $\mathrm{~ns}$ & $4,02^{*}$ \\
CFT & $\mathrm{ns}$ & $\mathrm{ns}$ & $\mathrm{ns}$ & $\mathrm{ns}$ & $\mathrm{ns}$ \\
Satisfação no trabalho & $\mathrm{ns}$ & $\mathrm{ns}$ & $\mathrm{ns}$ & $\mathrm{ns}$ & $\mathrm{ns}$ \\
Intenção de deixar a empresa & $\mathrm{ns}$ & $\mathrm{ns}$ & $3,83^{* *}$ & $\mathrm{~ns}$ & $4,30^{*}$ \\
Estresse & $\mathrm{ns}$ & $4,17 *$ & $\mathrm{~ns}$ & $\mathrm{~ns}$ & $7,94 * *$ \\
Absenteísmo & $\mathrm{ns}$ & $\mathrm{ns}$ & $\mathrm{ns}$ & $\mathrm{ns}$ & $9,00^{* *}$ \\
\hline
\end{tabular}

Nota. ns = não significativo.

Valores correspondem à estatística $\mathrm{F}(* \mathrm{p}<0,05 * * \mathrm{p}<0,01)$.

Com base nos resultados acima, foram incluídas variáveis dummy como controles nas análises das hipóteses previstas. Sexo e tamanho de empresa foram excluídos, por não apresentarem efeitos 
sobre as variáveis de interesse nessa amostra. Para a variável tempo de empresa, foi criada uma dummy, representando estar na empresa há mais de dez anos ( $0=$ até 10 anos; $1=$ mais de 10 anos). Permaneceram na análise as variáveis dummy cargo de chefia $(0=$ não;1=sim) e tipo de empresa (0=pública; 1 =privada).

Foram examinados diagramas dos resíduos e gráficos de probabilidade normal (Fox, 1991; Tabachnick \& Fidell, 2007) para avaliar as premissas da análise de regressão. Os resultados indicaram que as condições de normalidade, homocedasticidade, linearidade e independência dos resíduos não foram violadas, com exceção das variáveis absenteísmo e CFT. Ambas apresentaram distribuições assimétricas positivas e, portanto, foram submetidas a transformações logarítmicas, que foram eficazes na redução da assimetria. Nas análises subsequentes, as variáveis incluídas nos modelos correspondem ao logaritmo de CFT e ao logaritmo invertido do absenteísmo (Fox, 1991). Todas as análises realizadas foram acompanhadas pelo teste VIF, cujos resultados indicam não haver multicolinearidade entre os preditores incluídos nos modelos.

No que diz respeito à hipótese 1, relativa aos antecedentes do CTF (Tabela 3), o modelo 1, incluindo somente as variáveis de controle, não foi estatisticamente significativo. Entretanto, o modelo 2, que incluiu horário flexível, suporte gerencial, consequências na carreira e conflito família-trabalho, foi estatisticamente significativo, com coeficiente de determinação capaz de explicar $22 \%$ da variância do conflito trabalho-família $\left(\mathrm{R}^{2}=0,22, p<0,01\right)$. Tal efeito pode ser considerado de magnitude pequena a média ( $c f$. Cohen, Cohen, West, \& Aiken, 2002).

Tabela 3

\section{Regressão Múltipla: Antecedentes do Conflito Trabalho-Família}

\begin{tabular}{|c|c|c|c|c|c|c|}
\hline \multirow[t]{2}{*}{ Variáveis } & \multicolumn{3}{|c|}{ Modelo 1 - Controles } & \multicolumn{3}{|c|}{ Modelo 2 - Modelo Proposto } \\
\hline & $\beta$ & $\mathrm{t}$ & VIF & $\beta$ & $\mathrm{t}$ & VIF \\
\hline Idade & $-0,07$ & $-1,11$ & 1,21 & $-0,01$ & $-0,10$ & 1,25 \\
\hline Cargo de chefia & 0,12 & $2,09 *$ & 1,06 & 0,13 & $2,44^{*}$ & 1,07 \\
\hline Tempo de empresa & 0,04 & 0,58 & 1,20 & 0,03 & 0,52 & 1,23 \\
\hline Tipo de empresa & 0,09 & 1,53 & 1,08 & 0,14 & $2,47^{*}$ & 1,10 \\
\hline Horário flexível & & & & $-0,01$ & $-0,22$ & 1,08 \\
\hline Suporte gerencial & & & & $-0,23$ & $-3,79 * *$ & 1,37 \\
\hline Consequências na carreira & & & & 0,21 & $3,43 * *$ & 1,37 \\
\hline Conflito família-trabalho & & & & 0,18 & $3,40 * *$ & 1,05 \\
\hline$R^{2}$ & & 0,03 & & & $0,22 * *$ & \\
\hline$\Delta R^{2}$ & & & & & $0,19 * *$ & \\
\hline
\end{tabular}

Nota. $\mathrm{N}=296$

$* \mathrm{p}<0,05 * * \mathrm{p}<0,01$.

A análise dos parâmetros de cada variável de controle revela que, no modelo 1 , somente cargo de chefia apresentou relação estatisticamente significativa com CTF $(\beta=0,12 p<0,05)$. Dentre as variáveis consideradas na hipótese 1 , embora horário flexível (H1a) tenha se mostrado inversamente relacionada ao CTF, tal relação não foi estatisticamente significativa, ao contrário do verificado por Anderson et al. (2002). Esse resultado sugere que uma agenda de trabalho mais flexível não necessariamente minimiza o CTF. Nesse sentido, é possível considerar que uma rotina mais flexível não promove tranquilidade no trabalho e que as exigências profissionais podem continuar a interferir no cumprimento das demandas familiares. 
Por outro lado, foram confirmadas as demais relações propostas na hipótese 1, a saber: (a) suporte gerencial (H1b) apresenta-se inversamente relacionado ao CTF $(\beta=-0,23, p<0,01)$; (b) consequências na carreira (H1c) e CFT (H1d) estão positivamente associados com o CTF $(\beta=0,23$ e $\beta=0,18$, respectivamente, e $p<0,01$ para ambas), corroborando os achados de Anderson et al. (2002) e de Premeaux et al. (2007). Com relação ao suporte gerencial, as evidências mostram uma relação negativa entre esse construto e o CTF, indicando que o gestor imediato parece ter um papel importante na experiência de conflito vivida por seus subordinados. A percepção de consequências negativas sobre a carreira, que estaria associada a uma cultura organizacional intolerante com profissionais que buscam uma vida mais equilibrada, também teve impacto significativo sobre o CTF.

Quanto à hipótese 2, sobre os antecedentes do CFT (Tabela 4), o teste do modelo somente com as variáveis de controle não se mostrou significativo, mas a inclusão das variáveis propostas pela hipótese 2 (número de políticas de apoio aos dependentes, estrutura familiar e CTF) incrementou significativamente a variância explicada $\left(\Delta R^{2}=0,07, p<0,01\right)$. O coeficiente de determinação final foi capaz de explicar $10 \%\left(\mathrm{R}^{2}=0,10\right)$ da variância do $\mathrm{CFT}$, indicando um efeito de pequena magnitude $(c f$. Cohen et al., 2002).

Tabela 4

\section{Regressão Múltipla: Antecedentes do Conflito Família-Trabalho}

\begin{tabular}{lcccccc}
\hline Variáveis & \multicolumn{3}{c}{ Modelo 1 - Controles } & \multicolumn{3}{c}{ Modelo 2 - Modelo Proposto } \\
\hline & $\beta$ & $\mathrm{t}$ & $\mathrm{VIF}$ & $\beta$ & $\mathrm{t}$ & $\mathrm{VIF}$ \\
Idade & $-0,16$ & $-2,49^{*}$ & 1,21 & $-0,17$ & $-2,60^{* *}$ & 1,32 \\
Cargo de chefia & 0,01 & 0,21 & 1,06 & $-0,02$ & $-0,41$ & 1,10 \\
Tempo de empresa & 0,13 & $2,08^{*}$ & 1,20 & 0,11 & 1,86 & 1,21 \\
Tipo de empresa & $-0,01$ & $-0,15$ & 1,08 & $-0,03$ & $-0,51$ & 1,11 \\
Políticas de apoio utilizadas & & & & 0,04 & 0,62 & 1,07 \\
Estrutura familiar & & & & 0,08 & 1,35 & 1,17 \\
Conflito trabalho-família & & & & 0,24 & $4,18^{* *}$ & 1,04 \\
\hline $\boldsymbol{R}^{\mathbf{2}}$ & & 0,03 & & & 0,10 & \\
$\Delta \boldsymbol{R}^{\mathbf{2}}$ & & & & & $0,07 * *$ & \\
\hline
\end{tabular}

Nota. $N=296$

$* \mathrm{p}<0,05 * * \mathrm{p}<0,01$.

Ao analisar a significância estatística das variáveis de controle isoladamente, verifica-se que idade e tempo de empresa se mostraram significativamente associadas ao CFT. Com relação à hipótese 2a, não foi confirmada a relação negativa prevista entre o número de políticas de apoio aos dependentes usadas pelo empregado e o conflito família-trabalho, consistente com os resultados encontrados por Anderson et al. (2002), Judge e Colquitt (2004) e Premeaux et al. (2007). Também não houve suporte para a hipótese $2 \mathrm{~b}$, que previa uma relação positiva entre a complexidade da estrutura familiar e o CFT, ao contrário do verificado por Anderson et al. (2002) e por Premeaux et al. (2007). Dentre as variáveis propostas pela hipótese 2 , portanto, somente a variável CTF (H2c) mostrou-se positiva e significativamente relacionada com o CFT $(\beta=0,23, p<0,01)$.

Ainda com relação aos antecedentes dos conflitos trabalho-família e família-trabalho, houve suporte às hipóteses $1 \mathrm{~d}$, sobre o impacto positivo do CFT no CTF, e 2c, sobre o impacto positivo do CTF no CFT. Esses resultados são consistentes com a abordagem bidirecional do conceito, segundo a qual conflito trabalho-família e conflito família-trabalho representam construtos distintos que se influenciam mutuamente (Anderson et al., 2002; Eby et al., 2005). 
Sobre a hipótese 3 (Tabela 5), observou-se que quanto maior é o nível de conflito trabalhofamília (CTF) experimentado pelo profissional, menor é sua satisfação no trabalho. O teste do modelo proposto somente com as variáveis de controle mostrou-se significativo, capaz de explicar $5 \%$ $\left(\mathrm{R}^{2}=0,05, \mathrm{p}<0,01\right)$ da variância na satisfação no trabalho. A análise da significância estatística das variáveis de controle mostra que idade tem impacto positivo sobre a satisfação no trabalho $(p<0,05)$. A inclusão da variável CTF explicou adicionais $5 \%$ da variância $\left(\Delta R^{2}=0,05, p<0,01\right)$. Houve, portanto, suporte à hipótese 3 , uma vez que níveis mais altos de conflito trabalho-família (CTF) tendem a reduzir a satisfação no trabalho, indicando um efeito significativo, mas pequeno ( $c f$. Cohen et al., 2002).

Tabela 5

Regressão Múltipla: Satisfação no Trabalho

\begin{tabular}{lcccccc}
\hline Variáveis & \multicolumn{3}{c}{ Modelo 1 - Controles } & \multicolumn{3}{c}{ Modelo 2 - Modelo Proposto } \\
\hline & $\beta$ & $\mathrm{t}$ & $\mathrm{VIF}$ & $\beta$ & $\mathrm{t}$ & $\mathrm{VIF}$ \\
Idade & 0,14 & $2,22^{*}$ & 1,17 & 0,13 & $2,09^{*}$ & 1,17 \\
Cargo de chefia & 0,05 & 0,83 & 1,11 & 0,08 & 1,34 & 1,13 \\
Tempo de empresa & 0,10 & 1,66 & 1,20 & 0,10 & 1,62 & 1,20 \\
Tipo de empresa & 0,08 & 1,31 & 1,10 & 0,10 & 1,68 & 1,11 \\
Conflito trabalho-família & & & & $-0,23$ & $-3,99^{* *}$ & 1,03 \\
\hline $\boldsymbol{R}^{\mathbf{2}}$ & & $0,05^{* *}$ & & & $0,10^{* *}$ & \\
$\Delta \boldsymbol{R}^{\mathbf{2}}$ & & & & & $0,05^{* *}$ & \\
\hline
\end{tabular}

Nota. $N=296$

$* \mathrm{p}<0,05 * * \mathrm{p}<0,01$.

A hipótese 4 testou o efeito do nível de conflito trabalho-família (CTF) na intenção de deixar a empresa (Tabela 6). Tanto o modelo com variáveis de controle quanto o que considera a inclusão do CTF demostraram possuir significância estatística $(p<0,01)$. As variáveis de controle explicaram $6 \%$ $\left(\mathrm{R}^{2}=0,06\right)$ da variância da intenção de deixar a empresa, enquanto o CTF explicou $5 \%$ adicionais $\left(\Delta R^{2}=0,05\right)$, sinalizando um efeito de pequena magnitude (cf. Cohen et al., 2002).

Tabela 6

\section{Regressão Múltipla: Intenção de Deixar a Empresa}

\begin{tabular}{lcccccc}
\hline Variáveis & \multicolumn{3}{c}{ Modelo 1 - Controles } & \multicolumn{3}{c}{ Modelo 2 - Modelo Proposto } \\
\hline & $\beta$ & $\mathrm{t}$ & $\mathrm{VIF}$ & $\beta$ & $\mathrm{t}$ & $\mathrm{VIF}$ \\
Idade & $-0,13$ & $-2,08^{*}$ & 1,21 & $-0,11$ & $-1,86$ & 1,21 \\
Cargo de chefia & $-0,01$ & $-0,18$ & 1,06 & $-0,04$ & $-0,68$ & 1,08 \\
Tempo de empresa & $-0,13$ & $-2,09^{*}$ & 1,20 & $-0,14$ & $-2,28^{*}$ & 1,20 \\
Tipo de empresa & 0,08 & 1,42 & 1,08 & 0,06 & 1,09 & 1,09 \\
Conflito trabalho-família & & & & 0,23 & $4,05^{* *}$ & 1,03 \\
\hline $\boldsymbol{R}^{\mathbf{2}}$ & & $0,06^{* *}$ & & & $0,11^{* *}$ & \\
$\Delta \boldsymbol{R}^{\mathbf{2}}$ & & & & & $0,05^{* *}$ & \\
\hline
\end{tabular}

Nota. $\mathrm{N}=296$

$* \mathrm{p}<0,05 * * \mathrm{p}<0,01$. 
Entre as variáveis de controle, a idade e o tempo de empresa $(p<0,05)$ se mostraram associados à intenção de deixar a empresa, indicando que, na amostra estudada, quanto mais velho e com mais tempo de empresa estiver o profissional, menor tende a ser sua intenção de deixar a empresa. A variável CTF, proposta na hipótese 4 , mostrou ter um impacto pequeno, porém significativo $(\beta=0,23$, $p<0,01)$, indicando que um maior conflito trabalho-família está associado a uma maior intenção de deixar a empresa.

Quanto à hipótese 5 (Tabela 7), que propôs uma associação do nível de conflito trabalho-família (CTF) e do nível de conflito família-trabalho (CFT), com o estresse experimentado pelo profissional, observou-se que tanto o modelo com as variáveis de controle quanto o que considera a inclusão das variáveis propostas mostraram-se estatisticamente significativos $(p<0,01)$. As variáveis de controle explicaram 7\% $\left(\mathrm{R}^{2}=0,07 p<0,01\right)$ da variância do estresse, e os conflitos CTF e CFT explicaram adicionais $38 \%\left(\Delta R^{2}=0,38 p<0,01\right)$, indicando haver um efeito significativo e de considerável magnitude ( $c f$. Cohen et al., 2002).

A análise das variáveis de controle mostra que, de acordo com o modelo 1, a idade está negativamente associada ao estresse, enquanto que posição de chefia e tipo de empresa estão positiva e significativamente relacionados ao estresse. Como esperado, e corroborando a hipótese 5 , as variáveis CFT e CTF mostraram-se significativa e positivamente relacionadas ao estresse $(\beta=0,58$ e $\beta=0,15$ respectivamente, ambos com $p<0,01)$.

Tabela 7

\section{Regressão Múltipla: Estresse}

\begin{tabular}{|c|c|c|c|c|c|c|}
\hline \multirow[t]{2}{*}{ Variáveis } & \multicolumn{3}{|c|}{ Modelo 1 - Controles } & \multicolumn{3}{|c|}{ Modelo 2 - Modelo Proposto } \\
\hline & $\beta$ & $\mathrm{t}$ & VIF & $\beta$ & $\mathrm{t}$ & VIF \\
\hline 2. Idade & $-0,20$ & $-3,18 * *$ & 1,21 & $-0,13$ & $-2,75^{* *}$ & 1,24 \\
\hline 3. Cargo de chefia & 0,12 & $2,10^{*}$ & 1,06 & 0,05 & 1,12 & 1,08 \\
\hline 4. Tempo de empresa & 0,04 & 0,62 & 1,20 & 0,00 & $-0,04$ & 1,22 \\
\hline 6. Tipo de empresa & 0,12 & $2,09 *$ & 1,08 & 0,07 & 1,53 & 1,09 \\
\hline 7. Conflito trabalho-família & & & & 0,58 & $12,70^{* *}$ & 1,09 \\
\hline 8. Conflito família-trabalho & & & & 0,15 & $3,30 * *$ & 1,08 \\
\hline$R^{2}$ & & $0,07 * *$ & & & $0,45 * *$ & \\
\hline$\Delta R^{2}$ & & & & & $0,38 * *$ & \\
\hline
\end{tabular}

Nota. $\mathrm{N}=296$

$* \mathrm{p}<0,05 * * \mathrm{p}<0,01$.

Com relação à hipótese 6 , sobre a associação do nível de conflito família-trabalho (CFT) com o absenteísmo, observou-se na análise que tanto o modelo composto somente pelas variáveis de controle, quanto o que considera a inclusão da variável CFT, não apresentaram significância estatística no intervalo de confiança de $95 \%$ ( $R^{2}=0,03$ e 0,05 , respectivamente).

Em resumo, houve pleno suporte para as hipóteses a respeito das consequências do conflito trabalho-família. Um maior nível de CTF tende a reduzir a satisfação no trabalho (hipótese 3), a aumentar a intenção de deixar a empresa (hipótese 4) e a aumentar o estresse vivido pelo empregado (hipótese 5a). Esses resultados somam-se às evidências presentes na literatura sobre o tema (Anderson et al., 2002; Carr et al., 2008; Eby et al., 2005; Premeaux et al., 2007). Com relação às hipóteses sobre as consequências do conflito família-trabalho, as evidências mostraram que um maior nível de CFT tende a contribuir para o estresse (hipótese 5b), mas não para o absenteísmo (hipótese 6). Este último resultado mostrou-se diferente do obtido por Anderson et al. (2002), o que pode sinalizar uma distinção na forma como demandas familiares são administradas pelos grupos pesquisados. 


\section{Discussão e Conclusão}

O presente trabalho teve como objetivo examinar fatores associados aos conflitos entre vida pessoal e vida profissional, e suas consequências, em uma amostra de trabalhadores com nível superior, empregados em organizações de diferentes setores da economia, e que ocupam predominantemente cargos gerenciais. Esse tipo de empregado - tipicamente chamado de trabalhador do conhecimento (Drucker, 1999) - tende a ser de grande importância para organizações que buscam alta produtividade e que veem seus talentos como fonte de vantagens competitivas sustentáveis.

A pesquisa aqui relatada foi pioneira na avaliação empírica das proposições de Anderson et al. (2002) junto a uma amostra de trabalhadores brasileiros. Trata-se de um dos principais modelos sobre o tema encontrados na literatura internacional, que tem como diferencial a consideração de políticas formais e de práticas informais na vivência de conflitos, além do exame de suas consequências (Premeaux et al., 2007). Os resultados obtidos confirmam parcialmente sua pertinência no contexto brasileiro, o que representa uma importante contribuição teórica ao entendimento de um fenômeno complexo e de relevância crescente tanto para indivíduos e organizações - levando em conta as mudanças na estrutura das famílias e a maior demanda por resultados nas organizações -, quanto para a sociedade, visto que o conflito entre vida pessoal e vida profissional pode vir a afetar o equilíbrio familiar e a saúde dos trabalhadores.

Os resultados do estudo indicam que o conflito trabalho-família está associado à falta de suporte do superior imediato e à percepção de que a busca por um maior equilíbrio teria consequências negativas na carreira do profissional, fatores que representam práticas organizacionais informais, que não estão sob o controle direto da área de recursos humanos. Por outro lado, as evidências indicam que as políticas formais existentes, tipicamente desenvolvidas e promovidas pelo $\mathrm{RH}$, parecem ter pouco impacto sobre os conflitos: para essa amostra de trabalhadores, a flexibilidade de horário não contribuiu para minimizar o conflito trabalho-família e o uso de benefícios no cuidado com dependentes não teve efeito sobre o conflito família-trabalho. Em seu conjunto, essas evidências sugerem que a criação de uma cultura que valorize e respeite a vida pessoal dos trabalhadores tende a ser mais eficaz do que o desenvolvimento de políticas oficiais (Premeaux et al., 2007). Estas últimas podem, inclusive, ter efetividade limitada, caso os trabalhadores acreditem que seu uso implica em prejuízos ao desenvolvimento de sua carreira na empresa (McCarthy, Darcy, \& Grady, 2010).

Com relação à questão da flexibilidade, alguns autores vêm sugerindo que a adoção de horários de trabalho flexíveis por parte das empresas tem, na verdade, promovido a intensificação do trabalho (Kelliher \& Anderson, 2010), uma vez que a fragmentação de períodos de trabalho, embora conveniente, pode acabar levando o profissional a se ocupar em horários atípicos, como à noite, nos finais de semana e nos feriados (Rosso, 2008). Em linha com esse resultado, Premeaux et al. (2007) também não encontraram suporte para a hipótese de uma relação negativa entre autonomia no trabalho e conflito trabalho-família. A observação de que a flexibilidade de horário não reduz o conflito trabalho-família indica que essa política deve ser tratada com cautela, uma vez que a adoção de esquemas flexíveis pode ter consequências danosas que neutralizariam os benefícios apregoados para sua adoção. Sem dúvida, essa é uma área que carece de estudos mais profundos para avaliar as efetivas vantagens e desvantagens da flexibilidade.

No tocante às políticas de apoio ao cuidado com dependentes, que neste estudo parecem não contribuir para reduzir o conflito família-trabalho, é possível especular que, em seu estágio atual, estas tenham um espectro de cobertura relativamente limitado e/ou não sejam suficientemente efetivas para aliviar o profissional das demandas familiares que enfrenta. Uma pergunta aberta feita aos participantes desta pesquisa mostrou que os benefícios existentes tendem a ser pouco abrangentes e de ordem exclusivamente financeira. Auxílio-creche, auxílio-educação e planos de saúde e odontológico extensivos aos dependentes respondem por $70 \%$ do total de benefícios mencionados. Não desconsiderando a importância do suporte financeiro, esse pode ser insuficiente quando a pressão 
vivida pelo trabalhador é de outra natureza - como de tempo, por exemplo. Estudos futuros também podem clarificar esta questão.

A complexidade da estrutura familiar não teve impacto sobre o conflito família-trabalho. Apesar dessa variável ter sido medida de acordo com Anderson et al. (2002), talvez seja necessária uma revisão dessa métrica. A avaliação da complexidade familiar não leva em conta, por exemplo, a presença de filhos maiores de 18 anos. Porém, no caso da classe média brasileira, é comum a permanência de filhos para além desse limite, visto que estes continuam residindo com os pais e são financeiramente dependentes pelo menos até a conclusão do ensino superior. Estudos futuros poderiam relativizar a questão da maioridade civil dos filhos como critério para indicar menor complexidade, e explorar até que ponto fatores objetivos e subjetivos interferem na percepção de tal complexidade.

A ocorrência do conflito trabalho-família também parece associada ao conflito família-trabalho, corroborando outras evidências de que haveria uma relação mútua de causa e efeito entre CTF e CFT (Anderson et al., 2002; Eby el al., 2005). Nesse sentido, a existência de conflito em uma direção aumentaria a probabilidade de conflito na outra. Em outras palavras, quando as responsabilidades do trabalho passam a interferir no cumprimento das obrigações familiares, a incapacidade de atender plenamente aos compromissos familiares acabaria por promover interferências no trabalho, o que pode minar o bom andamento das atividades profissionais no longo prazo, provocando uma espiral viciosa de interferências negativas entre as demandas das duas dimensões.

Com relação às consequências dos conflitos, as evidências indicam que o conflito trabalhofamília reduz a satisfação no trabalho e aumenta a intenção do profissional de deixar a empresa. Além disso, o estresse também parece estar associado tanto ao CTF como ao CFT. Essas observações novamente confirmam a validade do modelo no tocante aos efeitos do conflito sobre atitudes e indicadores organizacionais. A exacerbação de demandas nos dois mundos, profissional e familiar, teria, portanto, consequências deletérias tanto sobre o bem-estar individual quanto sobre fatores associados ao desempenho das organizações. Nesse sentido, as empresas deveriam estar mais atentas às demandas excessivas que recaem sobre seus empregados, já que estariam correndo o risco de perder talentos importantes e ter seu desempenho comprometido.

Ainda sobre as consequências dos conflitos, não houve suporte para a relação entre conflito família-trabalho e absenteísmo, ao contrário do verificado por Anderson et al. (2002). Esse resultado pode estar associado aos arranjos familiares e ao suporte doméstico característicos do Brasil - que reduziriam a necessidade de o trabalhador se ausentar do trabalho em função de demandas familiares. As famílias estendidas ou famílias conviventes (Medeiros \& Osório, 2001), constituídas por um casal com filhos que habita a mesma residência que os pais de um dos cônjuges, são arranjos comuns no Brasil, onde haveria algum suporte para deixar filhos pequenos ou adoentados quando os pais precisam trabalhar. Mesmo em habitações distintas, a ajuda dos avós é uma fonte de suporte relativamente comum no Brasil (Dassen \& Braz, 2000). Adicionalmente, e em particular no que diz respeito à classe média brasileira, à qual pertencem os participantes da pesquisa, a presença de empregados domésticos (Sorj, Fontes, \& Machado, 2007) também costuma ser fonte de suporte. Nos Estados Unidos, onde a pesquisa de Anderson et al. (2002) foi conduzida, esse tipo de apoio tende a ser relativamente menor, e as creches prevalecem como alternativa para as famílias onde pai e mãe trabalham. Regras restritas à permanência da criança adoentada nesses ambientes imporiam com maior frequência aos pais que trabalham a necessidade de se ausentar. Futuras pesquisas poderiam investigar mais os efeitos do apoio doméstico, vindo de familiares ou de empregados, sobre a vivência do conflito em si e sobre suas consequências.

Vale ressaltar que, tomando como referência os dados observados na tabela de correlações, neste estudo o absenteísmo parece estar mais associado à insatisfação no trabalho do que às demandas familiares. Tendo em vista as exigências impostas ao trabalhador na atualidade (Oltramari et al., 2011; Rosso, 2008) e os riscos à manutenção do emprego decorrentes do absenteísmo, faltar ao trabalho pode não ser uma escolha para muitos trabalhadores no Brasil. No entanto, a presença do indivíduo na empresa não significa que o sofrimento advindo dos dilemas inerentes à dupla demanda deixe de ser 
experimentado. Ao contrário, é possível especular que o mal-estar seja até intensificado pela impossibilidade de atender a demandas familiares para comparecer ao trabalho. Além disso, estar presente fisicamente não significa que esse trabalhador esteja engajado em suas atividades profissionais, o que serve de alerta às organizações indiferentes ao conflito vivido por seus funcionários. Estudos futuros deveriam investigar também se o conflito família-trabalho tem implicações sobre o envolvimento e o desempenho dos trabalhadores.

Este estudo possui limitações que devem ser consideradas. Em função da ausência de controles experimentais, não é possível inferir causalidade. O número de participantes do estudo também constitui uma limitação, uma vez que impossibilitou o uso de ferramentas de análise estatística mais robustas, como os modelos estruturais. Além disso, não se pode descartar a possibilidade de que alguma variável preditora relevante tenha sido omitida, o que poderia afetar os estimadores. No entanto, o uso de controles para os efeitos de variáveis demográficas, como idade, cargo de chefia, tempo de empresa, e tipo de empresa, foram adotados para minimizar o problema. Embora a magnitude de alguns coeficientes de regressão tenha sido pequena, inviabilizando seu uso para fins preditivos, o fato de serem significantes permitiu verificar a maioria das hipóteses propostas, que era o objetivo central deste trabalho.

Como a amostra foi selecionada por conveniência e incluiu apenas profissionais com no mínimo nível superior completo, há restrições quanto à generalização dos resultados para profissionais em geral e para trabalhadores com diferentes níveis de instrução. Novas pesquisas poderiam verificar se os conflitos entre trabalho e família são vividos da mesma forma por profissionais com menor nível de escolaridade. Estudos futuros poderiam fazer recortes para melhor investigar a experiência de antecedentes e consequências dos conflitos em grupos específicos em termos demográficos e ocupacionais (mulheres; trabalhadores de empresas privadas). No presente estudo, também não foi avaliada a importância relativa do trabalho e da família para os trabalhadores, fator que pode agir como moderador das relações aqui investigadas (Bagger et al., 2008; Carr et al., 2008). Além disso, sugere-se que pesquisas de caráter qualitativo aprofundem o conhecimento sobre aspectos subjetivos relacionados aos conflitos, para melhor compreender como impactam, por exemplo, a satisfação no trabalho, a carreira e o bem estar do empregado. Tais estudos poderiam também investigar como as práticas organizacionais são percebidas pelos trabalhadores, bem como sua efetividade.

Em suma, o dilema entre o curto e o longo prazo aparece mais uma vez como uma questão crucial para as organizações. Se a empresa opta pelo foco no desempenho a curto prazo, tenderá a adotar medidas que ampliem a pressão sobre seus empregados e contribuam para que esses vivam mais intensamente os conflitos entre trabalho e família. Agindo dessa forma, porém, pode colocar em risco o desempenho e a retenção desses empregados a longo prazo, na medida em que essa pressão parece estar associada ao menor envolvimento e empenho do trabalhador, e a sua possível saída da empresa. Considerando-se que a amostra estudada corresponde a trabalhadores centrais para as organizações (Harvey, 2004), que tendem a receber significativos investimentos em treinamento e qualificação, as perdas decorrentes de sua saída podem ser expressivas - investimentos em capacitação não são recuperados, e o trabalhador ainda leva consigo todo o conhecimento adquirido sobre a empresa e sua dinâmica de funcionamento. Adicionalmente, tendo em vista que as novas gerações parecem buscar uma vida mais equilibrada, é possível que esse problema venha a se agravar com o tempo.

\section{Referências}

Albuquerque, L. G., \& Limongi-França, A. C. (1998). Estratégias de recursos humanos e gestão de qualidade de vida no trabalho: o stress e a expansão do conceito de qualidade total. Revista de Administração da Universidade de São Paulo, 33(2), 40-51. 
Anderson, S. E., Coffey, B. S., \& Byerly, R. T. (2002). Formal organizational initiatives and informal workplace practices: links to work-family conflict and job-related outcomes. Journal of Management, 28(6), 787-810. doi: 10.1016/S0149-2063(02)00190-3

Araújo, C., \& Scalon, C. (Org.). (2005). Gênero, família e trabalho no Brasil. Rio de Janeiro: Editora FGV.

Bagger, J., Li, A., \& Gutek, B. A. (2008). How much do you value your family and does it matter? The joint effects of family identity salience, family-interference-with-work, and gender. Human Relations, 61(2), 187-211. doi: 10.1177/0018726707087784

Bagtasos, M. R. (2011). Quality of work life: a review of literature. DLSU Business \& Economics Review, 20(2), 1-8.

Beauregard, T. A., \& Henry, L. C. (2009). Making the link between work-life balance practices and organizational performance. Human Resource Management Review, 19(1), 9-22. doi: 10.1016/j.hrmr.2008.09.001

Burke, R. (2009). Working to live or living to work: should individuals and organizations care? Journal of Business Ethics, 84(2), 167-172. doi: 10.1007/s10551-008-9703-6

Cappelli, P. (1999). The new deal at work: managing the market-driven workforce. Boston: Harvard Business School Press.

Carr, J. C., Boyar, S. L., \& Gregory, B. T. (2008). The moderating effect of work-family centrality on work-family conflict, organizational attitudes, and turnover behavior. Journal of Management, 34(2), 244-262. doi: 10.1177/0149206307309262

Cavazotte, F. S. N., Brollo, M. S., \& Moreno, V. A. (2009, setembro). Mobilidade computacional no trabalho: um estudo sobre as experiências dos usuários de telefones inteligentes. Anais do Encontro Nacional da Associação Nacional de Pós-Graduação e Pesquisa em Administração, São Paulo, SP, Brasil, 33.

Chesley, N. (2005). Blurring boundaries? Linking technology use, spillover, individual distress, and family satisfaction. Journal of Marriage and Family, 67(5), 1237-1248. doi: 10.1111/j.17413737.2005.00213.x

Cohen, J., Cohen, P., West, S., \& Aiken, L. (2002). Applied multiple regression/correlation analysis for the behavioral sciences (3rd ed.). Mahwah, NJ: Laurence Elrbaum.

Dassen, M. A., \& Braz, M. P. (2000). Rede social de apoio durante transições familiares decorrentes do nascimento de filhos. Psicologia: Teoria e Pesquisa, 16(3), 221-231. doi: 10.1590/S010237722000000300005

Drucker, P. F. (1999). Management challenges of the 21st century. New York: Harper Business.

Eby, L. T., Casper, W. J., Lockwood, A., Bourdeaux, C., \& Brinley, A. (2005). Work and family research in IO/OB: content analysis and review of the literature (1980-2002). Journal of Vocational Behavior, 66(1), 124-197. doi: 10.1016/j.jvb.2003.11.003

Ferreira, R. R., Ferreira, M. C., Antloga, C. S., \& Bergamaschi, V. (2009). Concepção e implantação de um programa de qualidade de vida no trabalho no setor público: o papel dos gestores. Revista de Administração da Universidade de São Paulo, 44(2), 147-157.

Fox, J. (1991). Regression diagnostics: an introduction (Sage University paper series on quantitative applications in the social sciences, $N^{\circ}$ 07-079). Newbury Park, CA: Sage. 
Furtado, L. M. G. P. (2012). Não há que ser flexível, há que ser forte: um estudo sobre a força dos limites no trabalho e na família (Dissertação de mestrado). Fundação Getúlio Vargas, Rio de Janeiro, RJ, Brasil.

Greenhaus, J. H. (2008). Innovations in the study of the work-family interface: introduction to the special section. Journal of Occupational \& Organizational Psychology, 81(3), 343-348. doi: 10.1348/096317908X332135

Greenhaus, J. H., \& Beutell, N. (1985). Sources of conflict between work and family roles. Academy of Management Review, 10(1), 76-88. doi: 10.5465/AMR.1985.4277352

Greenhaus, J. H., Collins, K. M., \& Shaw, J. D. (2003). The relation between work-family balance and quality of life. Journal of Vocational Behavior, 63(3), 510-531. doi: 10.1016/S00018791(02)00042-8.

Greenhaus, J. H., \& Powell, G. N. (2006). When work and family are allies: a theory of work-family enrichment. Academy of Management Review, 31(1), 72-92. doi: 10.5465/AMR.2006.19379625

Hair, J. F., Jr., Anderson, R. E., Tatham, R. L., \& Black, W. C. (1998). Multivariate data analysis (5th ed.). New Jersey: Prentice-Hall.

Harvey, D. (2004). Condição pós-moderna (13a ed.). São Paulo: Edições Loyola.

Judge, T. A., \& Colquitt, J. A. (2004). Organizational justice and stress: the mediating role of workfamily conflict. Journal of Applied Psychology, 89(3), 395-404. doi: 10.1037/00219010.89.3.395

Kelliher, C., \& Anderson, D. (2010). Doing more with less? Flexible working practices and the intensification of work. Human Relations, 63(1), 83-106. doi: 10.1177/0018726709349199

Lewis, S., \& Cooper, C. L. (1999). The work-family research agenda in changing contexts. Journal of Occupational Health Psychology, 4(4), 382-393. doi: 10.1037/1076-8998.4.4.382

McCarthy, A., Darcy, C., \& Grady, G. (2010). Work-life balance policy and practice: understanding line manager attitudes and behaviors. Human Resource Management Review, 20(2), 158-167. doi: 10.1016/j.hrmr.2009.12.001

McQuitty, S. (2004). Statistical power and structural equation models in business research. Journal of Business Research, 57(2), 175-183. doi: 10.1016/S0148-2963(01)00301-0

Medeiros, M., \& Osório, R. (2001). Arranjos domiciliares e arranjos nucleares no Brasil: classificação e evolução de 1977 a 1998 [Texto para discussão, Nº 788]. Brasília, DF: IPEA.

Oliveira, P. M., \& Limongi-França, A. C. (2005). Avaliação da gestão de programas de qualidade de vida no trabalho. RAE-eletrônica, 4(1). Recuperado de http://www.scielo.br/pdf/raeel/v4n1/v4n1a05.pdf. doi: 10.1590/S1676-56482005000100005

Oltramari, A. P., Grisci, C. L. I., \& Weber, L. (2011). Carreira e relações familiares: dilemas de executivos bancários. Revista Mal-estar e Subjetividade, 11(1), 101-133.

Pochmann, M. (2002). O trabalho sob fogo cruzado: exclusão, desemprego e precarização no final do século XX (3a ed.). São Paulo: Contexto.

Powell, G. N., \& Greenhaus, J. H. (2010). Sex, gender, and the work-to-family interface: exploring negative and positive interdependencies. Academy of Management Journal, 53(3), 513-534. doi: 10.5465/AMJ.2010.51468647 
Premeaux, S. F., Adkins, C. L., \& Mossholder, K. W. (2007). Balancing work and family: a field study of multi-dimensional, multi-role work-family conflict. Journal of Organizational Behavior, 28(6), 705-727. doi: 10.1002/job.439

Rosso, S. D. (2008). Mais trabalho! A intensificação do labor na sociedade contemporânea. São Paulo: Boitempo.

Sant'Anna, A. S., \& Kilimnik, Z. M. (Orgs.). (2011). Qualidade de vida no trabalho: abordagens e fundamentos. Rio de Janeiro: Elsevier; Belo Horizonte: Fundação Dom Cabral.

Santos, H. B., \& Casado, T. (2011, setembro). O tradicional reconfigurado: a proposta de um modelo para casais de dupla carreira. Anais do Encontro Nacional da Associação Nacional de PósGraduação e Pesquisa em Administração, Rio de Janeiro, RJ, Brasil, 35.

Siqueira, M. M. M. (2008). Satisfação no trabalho. In M. M. M. Siqueira (Org.), Medidas do comportamento organizacional (pp. 265-274). Porto Alegre: Artmed.

Sorj, B., Fontes, A., \& Machado, D. C. (2007). Políticas e práticas de conciliação entre família e trabalho no Brasil. Cadernos de Pesquisa, 37(132), 574-594. doi: 10.1590/S010015742007000300004

Tabachnick, B. G., \& Fidell, L. S. (2007). Using multivariate statistics (5th ed.). Boston: Pearson Education, Inc./Allyn and Bacon.

Tanure, B., Carvalho, A., Neto, \& Andrade, J. (2007). Executivos: sucesso e (in)felicidade. Rio de Janeiro: Elsevier.

Walton, R. E. (1973). Quality of working life: what is it? Sloan Management Review, 15(1), 11-21.

Yasbek, P. (2004). The business case for firm-level work-life balance policies: a review of the literature. Wellington, NZ: Department of Labor. Recuperado de http://www.dol.govt.nz/publication-view.asp?ID=191 